\title{
MRI Monitoring of Cerebral Blood Flow after the Delivery of Nanocombretastatin across the Blood Brain Tumor Barrier
}

Sunalee Gonawala1, Madhava Aryal², James R. Ewing ${ }^{2}$, Ana C. deCarvalho', Steven Kalkanis ${ }^{1}$ and Meser M. Ali*

${ }^{1}$ Department of Neurosurgery, Cellular and Molecular Imaging Laboratory, Henry Ford Hospital, USA

${ }^{2}$ Department of Neurology, Henry Ford Hospital, Detroit, MI 48202, USA

\begin{abstract}
Introduction of polymeric nanoparticles in cancer therapeutics is widely investigated since nanomedicine often enables the intratumoral delivery of drugs with increased efficacy with minimal side effects. In this study MRI monitoring was employed to study the therapeutic effect of nanocombretastatin (G3-CA4) in an orthotopic glioma model. Water insoluble combretastatin (CA4) was conjugated to a small-sized water soluble G3-succinamic acid PAMAM dendrimer. Nanoconstruct sizes were determined by TEM to be 3 to $5 \mathrm{~nm}$. Intravenous (i.v.) delivery of G3-CA4 in an orthotopic glioma model produced a long-lived ischemia accompanied by necrosis at the core of the tumor but leaving a rim of viable tissue. In contrast, delivery of CA4 alone has no therapeutic effect in an experimental rat model of glioma.
\end{abstract}

Keywords: MRI; Dendrimer; Nanocombretastatin; Cerebral blood flow; Brain tumor

\section{Introduction}

Glioma is a malignant central nervous system neoplasm that is highly vascular and resistant to all current therapies. High-grade gliomas, while relatively rare [1,2] are almost invariably fatal; even given best therapy, the median survival is about 15 months [3]. These tumors have proven very difficult to treat. Cancer treatment usually begins with surgery followed by radiation or chemotherapy [4].

Increased vascularization and angiogenesis are fundamental to glioma cell survival and proliferation [5]. Malignant gliomas are typically characterized by a discharge of vascular endothelial growth factor (VEGF), which is a crucial regulator and promoter of angiogenesis [6]. As a result, anti-angiogenic remedies targeting VEGF or VEGF receptors (VEGFRs) were proposed as effective tools for regulating the growth of malignant gliomas. However, early clinical trials using humanized monoclonal antibodies against small-molecule tyrosine kinase inhibitors targeting different VEGFRs and VEGF (Bevacizumb) alone or together with other therapeutic agents showed mixed results [6-8]; most reports indicate the gliomas developed resistance to antiangiogenic medications [7-10].

Alternative therapies known as Tumor-Vascular Disrupting Agents (T-VDAs) diverge from anti-angiogenic strategies and directly target established tumor vasculature. T-VDAs selectively disrupt the immature and rapidly proliferating endothelial cells $[11,12]$ of established tumor vasculature by direct apoptosis or by effects associated to the endothelial cell's reliance on a tubulin cytoskeleton to preserve cell shape. These agents block the blood flow in tumors, resulting in an ischemia leading to a cascade of secondary tumor cell death in the tumor core $[13,14]$. T-VDAs can have drastic effects on the three dimensional shape of freshly formed endothelial cells, with slight or no consequence on quiescent endothelial cells $[14,15]$ because newly formed endothelial cells are more sensitive to treatment than mature cells, since the former lack a fully developed actin cytoskeleton that maintains cell shape despite depolymerization of the tubulin cytoskeleton [16,17]. The most promising T-VDA is combretastatin (CA4), [18-20] though watersolubility poses a major challenge. Combretastatin phosphate (CA4-P) which is a water-soluble form of CA4 has been synthesized to improve bioavailability. CA4-P also has the ability to disrupt cell-cell contacts between endothelial cells mediated by the vascular endothelial (VE)cadherin/ $\beta$-catenin complex [21]. However, the presence of smooth muscle cells, a characteristic feature of normal tissue vasculature inhibits this disruption [22]. Targeting newly formed endothelial cells in immature or abnormal vessels that lack a full complement of smooth muscle or pericyte support is believed to contribute to drug specificity $[14,17]$. Although CA4 can cause necrosis in the tumor core of various types of solid tumors [17,23], in gliomas CA4 alone does not effectively induce a central necrosis.

Tumor vasculature disruption approaches are suitable for nanoparticle-based therapeutics because they do not require the drug to be delivered to every cell in the tumor. We hypothesize that nanoformulation of CA4 may represent an improved approach to target tumor vasculature of glioma. The typically high levels of angiogenic activity in brain tumors results in the formation of abnormally dilated vessels with fenestration and leaky inter-endothelial gaps [24-26]. In this hyper-permeable vasculature nanoparticles can extravasate and be preferential retained in the tumor interstitium following systemic administration, a phenomenon known as the Enhenced Permeability and Retention (EPR) effect [27] albeit the effective transvascular delivery of nanoparticles through the blood-brain tumor barrier (BBTB) of malignant gliomas remains a challenge. A recent study to evaluate the effect of nanoparticle size on BBTB permeability demonstrated that gadolinium chelated dendrimer nanoparticles with core sizes less than $12 \mathrm{~nm}$ penetrated the BBTB, whereas larger nanoparticles were restricted [27-30]. Spherical nanoparticles with core sizes between 4 to $10 \mathrm{~nm}$ that are smaller than the upper limit of pore size in the BBTB retained peak blood concentrations for several hours [27,31,32]. Dendrimer-based paramagnetic nanoparticles that can preferentially accumulate in an orthotopic preclinical glioma model were recently developed $[26,33]$.

${ }^{*}$ Corresponding author: Meser M. Ali, Department of Neurosurgery, Cellular and Molecular Imaging Laboratory, Henry Ford Hospital, 1 Ford Place, 2F, Box 82 Detroit, MI 48202, USA, Tel: 3138744479; Fax: 3138744494; E-mail: mali8@hfhs.org

Received: October 11, 2018; Accepted: October 22, 2018; Published: October 25 2018

Citation: Gonawala S, Aryal M, Ewing JR, DeCarvaloh CA, Kalkanis S, et al. (2018) MRI Monitoring of Cerebral Blood Flow after the Delivery of Nanocombretastatin across the Blood Brain Tumor Barrier. J Nanomed Nanotechnol 9: 516. doi: 10.4172/2157-7439.1000516

Copyright: (c) 2018 Gonawala S, et al. This is an open-access article distributed under the terms of the Creative Commons Attribution License, which permits unrestricted use, distribution, and reproduction in any medium, provided the original author and source are credited. 
Citation: Gonawala S, Aryal M, Ewing JR, DeCarvaloh CA, Kalkanis S, et al. (2018) MRI Monitoring of Cerebral Blood Flow after the Delivery of Nanocombretastatin across the Blood Brain Tumor Barrier. J Nanomed Nanotechnol 9: 516. doi: 10.4172/2157-7439.1000516

Page 2 of 7

While there are nanoformulations of CA4 developed for the distribution of the active form of CA4 to tumors [34], their sizes have been significantly larger than $12 \mathrm{~nm}$, the upper limit of pore size in the BBTB $[27,31]$. With this in mind, we proposed to use CA4 conjugated to our recently developed dendrimer-based nanoparticle (G3-CA4) with nanoscale diameters ( 3 to $8 \mathrm{~nm}$ ) via a hydrolytically labile ester linkage for the development of an effective brain drug delivery system, and to follow the delivery and action of this nanoparticle with noninvasive MR imaging. We hypothesized that the EPR effect of these dendrimer-based G3-CA4 would lead to an accumulation in brain tumors and induce necrosis at the primary GBM core.

\section{Materials and Methods}

All reagents used were purchased from Sigma Aldrich (St. Louis, MO) unless otherwise stated. G3-PAMAM succinamic acid (10 wt. \%) in water was purchased from Sigma Aldrich and dried in a freeze dryer prior to use. 4-Dimethylaminopyridine (99\%), Dimethylsulfoxide anhydrous ( $\geq 99.9 \%)$, N,N'-dicyclohexylcarbodiimide (99\%) and Combretastatin-A4 ( $\geq 99$ ) were used without further purifications. Dendrimeric combretastatin conjugates were purified by repeated ultrafiltration with deionized water using appropriate molecular weight cut-offs (Millipore's Amicon Ultra centrifugal filters). ${ }^{1} \mathrm{H}$ - NMR spectra were obtained using a VARIAN MR 400 NMR spectrophotometer. Deuterated DMSO (DMSO- $\mathrm{d}_{6}$ ) was used as NMR solvent. Chemical shifts $(\delta)$ are given in ppm with reference to the internal standard Tetramethylsilane (TMS, $\delta=0 \mathrm{ppm}$ ). The molecular weights of the conjugates were analyzed using ESI-Mass or MALDI-TOF spectrometry.

\section{Synthesis of G3-PAMAM-(Succinamic acid)-Combretastatin ${ }_{26}$ conjugate}

To a solution of G3-PAMAM-(succinamic acid) ${ }_{32}(0.15 \mathrm{~g}, 0.015$ mmol, 1 equiv.) dissolved in DMSO $(15 \mathrm{ml})$, combretastatin-A4 (CA4) ( $0.15 \mathrm{~g}, 0.48 \mathrm{mmol}, 32$ equiv.) dissolved in DMSO $(10 \mathrm{ml})$ was added. Then to the above reaction mixture, 4-dimethylaminopyridine $(1.0 \mathrm{mg})$ and $\mathrm{N}, \mathrm{N}$-Dicyclohexylcarbodiimide $(0.099 \mathrm{~g}, 0.48 \mathrm{mmol}$, 32 equiv.) were added and the reaction was stirred for $24 \mathrm{~h}$ at room temperature. After $24 \mathrm{~h}$, the reaction mixture was transferred to a 500 $\mathrm{ml}$ Erlenmeyer flask, diluted with distilled water $(400 \mathrm{ml})$ and stirred at room temperature for 0.5 hour. After this, the reaction mixture was left in refrigerator overnight to allow the precipitate to settle. The resultant clear solution was filtered through $0.45 \mu \mathrm{m}$ filter unit. Finally, the filtrate was diafiltered using Amicon ${ }^{\circ}$ Ultra centrifugal filter $(3,000$ MWCO, regenerated cellulose) and was washed with distilled water (3 times) under the same conditions. The sample was then pre-frozen in liquid nitrogen and freeze dried. Finally, $0.16 \mathrm{~g}$ of white product was obtained. MALDI-TOF (Figure S1) m/z: $18500 \mathrm{~g}$.

\section{Animal model}

Animal studies were performed adhering to approved procedures of the Institutional Animal Care and Use Committee of Henry Ford Hospital, Detroit, MI. U-251 human glioma cells were provided by Dr. Ana C. deCarvalho, Henry Ford Hospital. These cells were cultured in $75 \mathrm{~cm}^{2}$ tissue culture flasks using Dulbecco's modified Eagle's medium (DMEM) supplemented with 10\% fetal bovine serum (FBS), penicillin $(100 \mathrm{IU} / \mathrm{mL})$, and streptomycin $(100 \mu \mathrm{g} / \mathrm{mL})$ until they were $80-90 \%$ confluent. The cells were collected by trypsinization, washed and centrifuged to make a cell suspension of $4 \times 10^{5}$ cells/ $5 \mu \mathrm{L}$.

Six- to eight-week-old athymic nude rats weighing 150-170 g (Charles River Laboratory, Inc.) were anesthetized by intraperitoneal injection of a mixture of $100 \mathrm{mg} / \mathrm{kg}$ ketamine and $15 \mathrm{mg} / \mathrm{kg}$ xylazine. Each rat was placed on a stereotactic head holder (Kopf, Cayunga, CA), the surgical zone was shaved and swabbed with betadine solution, and the eyes were coated with Lacri-lube (Allergan Inc., Irvine CA). After draping, a $1-\mathrm{cm}$ incision was made $2 \mathrm{~mm}$ to the right of the midline, $1 \mathrm{~mm}$ retro-orbitally and the skull was exposed with cotton-tip applicators. An HP-4 dental drill bit was used with a micromanipulator to drill a hole $3 \mathrm{~mm}$ to the right and $1 \mathrm{~mm}$ anterior to the bregma, with care not to penetrate the dura. A \#2701 $10 \mu \mathrm{L}$ Hamilton syringe with a \#4 point, $26 \mathrm{~s}$ gauge-needle containing $4 \times 10^{5} \mathrm{U}-251$ tumor cells in 5 $\mu \mathrm{L}$ was lowered to the depth of $3.5 \mathrm{~mm}$, then raised to the depth of 2.5 $\mathrm{mm}$. The cells were injected stepwise at a rate of $0.5 \mu \mathrm{L} / 30 \mathrm{sec}$ until the entire volume was injected. During and after the injection, careful note was made for any reflux from the injection site. Two to three minutes after completing the injection, the syringe was withdrawn in a stepwise manner. The surgical hole was sealed with bone wax. Finally, the skull was swabbed with betadine before suturing the skin over the injection site. The orthotopic tumor was allowed to grow for 4 to 5 weeks to a diameter of 3 to $5 \mathrm{~mm}$.

\section{G3-PAMAM-(Succinamic acid)-Combretastatin pharmacokinetics in serum: LC-MS/MS method for determination of CA4 in serum}

The concentrations of CA4 in serum were estimated by mass spectrometric detection (LC-MS/MS). To $100 \mu \mathrm{l}$ of plasma, $200 \mu \mathrm{l}$ methanol containing $2 \%$ formic acid was added, and the mixture was vortex-mixed for 1 minute, then centrifuged at 14000 RPM for 2 minutes. Zileuton was used as internal standard. The supernatant was collected, and a volume of $10 \mu \mathrm{l}$ was injected into the HPLC. Chromatographic analysis was performed using a Waters Model 2695 separations system (Milford, MA, USA) as described in our previous report [35].

\section{In vivo MRI and measurement of Cerebral blood flow (CBF)}

All MRI studies were performed using a $21 \mathrm{~cm}$ bore, 7 Tesla magnet and a Bruker spectrometer and console running Paravision 6.0.1. Gradient maximum strengths and rise times were $250 \mathrm{mT} / \mathrm{m}$ and 120 $\mu$ s. All MRI image sets were acquired with a $32 \times 32 \mathrm{~mm}^{2} \mathrm{FOV}$. The transmit coil was a RAPID (RAPID MR International, Columbus, $\mathrm{OH}$ ) Quadrature Birdcage coil; the receive coil was RAPID two-channel phased-array surface coil for rat brain imaging.

About 22 days after tumor implantation each animal was anesthetized ( $0.8 \%$ to $1.0 \%$ halothane) and a $26 \mathrm{~g}$ dental catheter was inserted into a tail vein to allow the injection of contrast agent. The animal was then placed in the magnet bore. Core temperature was controlled at $\left(37^{\circ} \mathrm{C}\right.$ $\pm 1^{\circ} \mathrm{C}$ ) using a warm-air supply. Arterial spin labeling (ASL), pre- and post-contrast $\mathrm{T}_{1}$-weighted, pre-contrast $\mathrm{T}_{2}$-weighted, and pre-contrast apparent diffusion coefficient (ADC) image sets were acquired. Prior to the second $\mathrm{T}_{1}$-weighted image, a bolus injection of the CA (Magnevist, Bayer Healthcare Pharmaceuticals, Wayne, New Jersey), $0.25 \mathrm{mmol} / \mathrm{kg}$ at undiluted concentration, no flush, was performed by hand push.

Tissue perfusion was imaged using arterial spin labeling MRI (ASLMRI) using a fast spin-echo acquisition [36]. Perfusion imaging time was $\sim 13$ min with the following parameters: TR $=1500 \mathrm{~ms}, 16$ Segments, 4 echoes per segment, echo spacing $=12.02 \mathrm{~ms}, \operatorname{NEX}=8$, Matrix $=128 \mathrm{x}$ 64 , Spectral Width $=25 \mathrm{kHz}, \mathrm{FOV}=32 \mathrm{~mm}$, Slice $=1 \mathrm{~mm}$. After a locater image, high-resolution $\mathrm{T}_{2}$, and a pre-treatment flow sequence was run, and then either CA4 $(50 \mathrm{mg} / \mathrm{kg})$ or G3-CA4 $(50 \mathrm{mg} / \mathrm{kg}$, equivalent to CA4 dose) was administered via an indwelling catheter. 
Citation: Gonawala S, Aryal M, Ewing JR, DeCarvaloh CA, Kalkanis S, et al. (2018) MRI Monitoring of Cerebral Blood Flow after the Delivery of Nanocombretastatin across the Blood Brain Tumor Barrier. J Nanomed Nanotechnol 9: 516. doi: 10.4172/2157-7439.1000516

Page 3 of 7

Prior to drug administration, a pulsed gradient spin-echo DWI sequence was run in three directions $(x, y, z)$ to generate a parametric map of the trace of ADC. DWI sequence parameters were as follows: matrix $128 \times 64,13$ slices, $0.8 \mathrm{~mm}$ thickness, $0.2 \mathrm{~mm}$ gap, repetition time $(\mathrm{TR})=1500 \mathrm{~ms}$, echo time $(\mathrm{TE})=40 \mathrm{~ms}$, number of echoes $(\mathrm{NE})$ $=1, \mathrm{~b}$-values $=0,600,1217 \mathrm{~s} / \mathrm{mm}^{2}$, gradient amplitude $=107 \mathrm{mT} / \mathrm{m}$, gradient duration $=10 \mathrm{~ms}$ ).

Two high-resolution $\mathrm{T}_{1}$-weighted spin-echo images were acquired pre- and post-CA, to locate the tumor and its size, with the following parameters: $\mathrm{FA}=45^{\circ}, 180^{\circ}$, matrix $256 \times 192,27$ slices, $0.4 \mathrm{~mm}$ thickness, $0.1 \mathrm{~mm}$ gap, $\mathrm{NE}=1, \mathrm{NA}=4$, TE/TR $=16 / 800 \mathrm{~ms}$. A highresolution $\mathrm{T}_{2}$-weighted spin-echo image was acquired pre-contrast with the following parameters: $\mathrm{FA}=90^{\circ}, 180^{\circ}$, matrix $256 \times 192,27$ slices, $0.4 \mathrm{~mm}$ thickness, $0.1 \mathrm{~mm}$ gap, $\mathrm{NE}=4, \mathrm{NA}=2, \mathrm{TE} / \mathrm{TR}=(20,40,60$, 80)/3000 ms).

\section{Histopathology}

Tissues were stained for red with vWF-TRITC and green for FITCtomato lectin delineating endothelial lining and blue for nuclear dapi using standard immune histochemical procedure as described in our previous report [36].

\section{Results and Discussion}

A
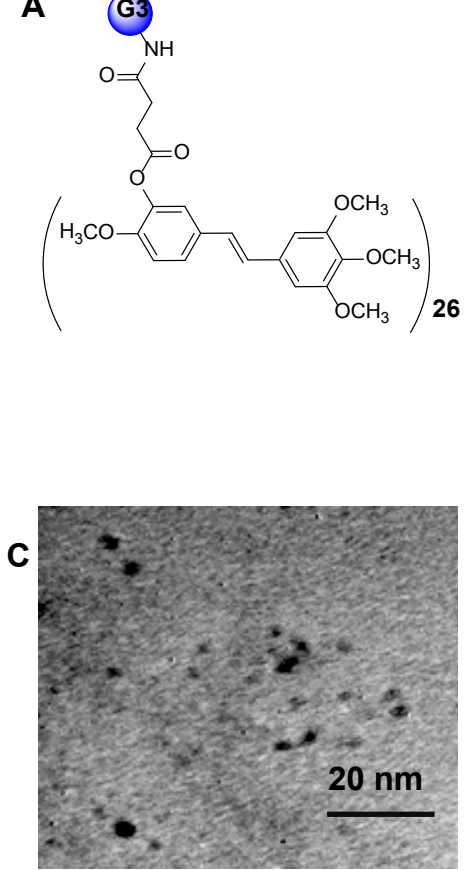

\section{Synthesis}

The DMAP

(4-dimethylaminopyridine)/DCC (dicyclohexylcarbodiimide) coupling method was used to conjugate CA4 with G3-succinamic acid dendrimer (G3-COOH) ${ }_{32}$. The G3CA4 conjugate (Figure 1A) was purified by diafiltration using a C-3 membrane filter (MWCO 3000) and characterized by ${ }^{1} \mathrm{H}-\mathrm{NMR}, \mathrm{UV}$ visible, and MALDI-TOF mass spectrometric methods. The ${ }^{1} \mathrm{H}-\mathrm{NMR}$ spectrum Figure 1B showed multiplets between $\delta 1.0$ and $4.0 \mathrm{ppm}$ corresponding to the presence of $-\mathrm{CH}$ and $-\mathrm{CH}_{2}$ protons from $\mathrm{G} 3$ PAMAM-succinamic acid and CA4. The peaks between $\delta 6.4$ and 8.4 ppm correspond to the presence of hydrogen atoms on the phenyl rings in CA4 [37]. The peak at $\delta 8.9 \mathrm{ppm}$ in CA4 that corresponds to $-\mathrm{OH}$ is absent in the resulted G3-CA4 conjugated product due to the conjugation with G3-succinamic acid dendrimer. Due to the overlapping of the multiple peaks in the NMR spectrum, a complete characterization of the proton spectra was not achieved.

The number of CA4 molecules per dendrimer was estimated using MALDI-TOF mass spectroscopy. G3- succinamic acid (G3-COOH) has a mass of $10,111 \mathrm{~g} /$ mole and G3-CA4 conjugate was shown to have a mass of $18,500 \mathrm{~g} / \mathrm{mole}$ (Figure S1). Since CA4 has an approximate mass of $316.34 \mathrm{~g}$, the conjugate was shown Figure 1A to contain 26 CA4 molecules per dendrimer corresponding to a conjugation yield of

G3-CA4 Conjugate

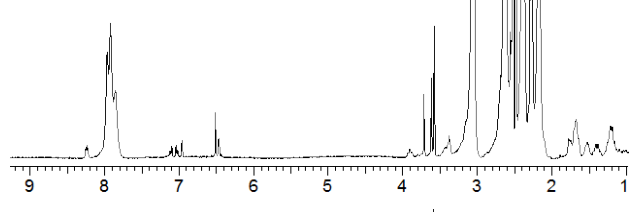

CA4

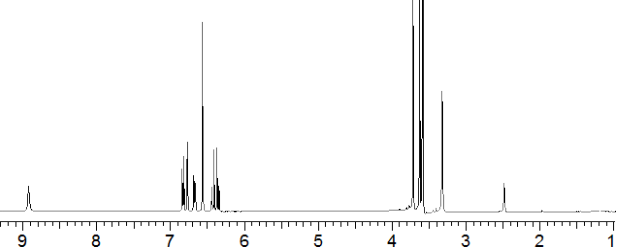

G3PAMAM-Succinimic acid

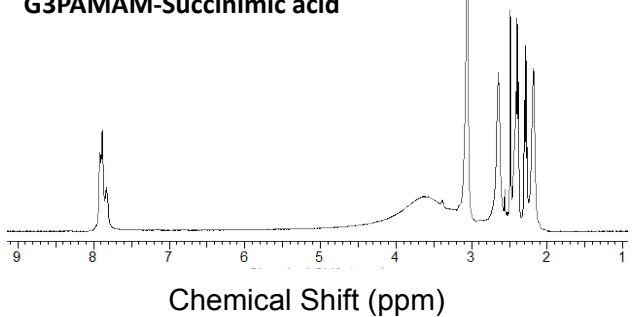

Figure 1: Schematic view of CA4 conjugated with a G3 PAMAM succinamic acid dendrimer (A). ${ }^{1} \mathrm{H}$ NMR spectrum of G3-(CA4) ${ }_{26}$, CA4, and G3 PAMAM succinamic acid in DMSO (B), TEM image of G3-CA4 conjugate (C). 
Citation: Gonawala S, Aryal M, Ewing JR, DeCarvaloh CA, Kalkanis S, et al. (2018) MRI Monitoring of Cerebral Blood Flow after the Delivery of Nanocombretastatin across the Blood Brain Tumor Barrier. J Nanomed Nanotechnol 9: 516. doi: 10.4172/2157-7439.1000516

Page 4 of 7

$81 \%$. The size of G3-CA4 conjugate was estimated to be approximately 3 to $5 \mathrm{~nm}$ using transmission electron microscope (TEM) images as shown in Figure 1C. The G3-CA4 polymer conjugate (Figure 1A) was highly water soluble, while natural free CA4 was water insoluble [38]. Therefore, we succeeded in conjugating a water insoluble CA4 with highly soluble G3 polymers that bear an additional advantage as a procirug with enhanced aqueous solubility which in turn increase the bioavailability of the drug that will result in enhanced cytotoxic activity.

\section{Absorption spectra}

The absorbance spectra of CA4, G3-succinamic acid, and G3CA4 conjugate (in $\mathrm{H}_{2} \mathrm{O}$ ) is shown in Figure 2. Only G3-CA4 showed an absorption maxima $\left(\lambda_{\max }\right)$ around $325 \mathrm{~nm}$ and $280 \mathrm{~nm}$. Like G3Cur, conjugation of CA4 to G3 dendrimer did not shift the absorption maxima of free CA4 [35]. Free CA4 in DMSO was used to generate a standard curve for the estimation of CA4 content in G3-CA4 conjugate as shown in Figure S2.

\section{Pharmacokinetics of the G3-CA4}

All animal experiments were approved by the Institutional Animals Care and User Committee (IACUC). We determined serum pharmacokinetics of the G3-CA4. In order to perform bioavailability of G3-CA4, the rats were randomly assigned to either G3-CA4 or CA4 group. G3-CA4 (100 mg/kg equivalent to CA4 in water) was administered to rats intravenously. The rats were anesthetized at $0,0.25,0.5,1,2,3$ and 4 hours later, blood was collected by femoral catheterization, with the serum separated by centrifugation at 14000 rpm for $2 \mathrm{~min}$. HPLC profiles of serum are depicted in Figure S3. 3 rats were used for each time point. A maximum serum concentration of $31 \mu \mathrm{M}$ of G3-CA4 per $\mathrm{mL}$ was detected at $0.25 \mathrm{~h}$ after intravenous administration of G3-CA4. LC/MS/MS method for the detection and quantification of free CA4 in rat serum was also performed, and the amount of free CA4 in the serum was not detectable. Therefore, conjugation of CA4 to a G3 dendrimer resulted an improved bioavailability of CA4. However, CA4 levels in the serum reported was as CA4 released from G3-CA4 conjugate per milliliter of serum.

\section{In vivo MRI monitoring Cerebral Blood Flow in U251 cerebral tumors after treatment with CA4 and G3-CA4}

Tumor blood flow differs from that of normal tissue [14], particularly in its lack of regulation. This leads to a vulnerability that can be exploited by agents like CA4 which weaken tubulin in the microvessels of the tumor, leading to the collapse of the microvessels and failure of perfusion in the tumor core.

Nude rats $(\mathrm{n}=2)$ with intracerebral U251 tumors were studied 20 days after implantation. After a locator image, high-resolution $\mathrm{T}_{2}, \mathrm{ADC}$, and two pre-treatment flow studies were acquired. Following this, either CA4 $(50 \mathrm{mg} / \mathrm{kg}$ ) or G3-CA4 (50 mg/kg, equivalent to CA4 dose) was administered via an indwelling catheter. Five more flow studies were conducted at $\sim 20 \mathrm{~min}$ intervals followed by contrast agent injection and a post-contrast $\mathrm{T}_{1}$-weighted study. The animal was then removed from the magnet. Twenty-four hours later, the $\mathrm{T}_{1}, \mathrm{~T}, \mathrm{ADC}$, and two further flow studies were conducted. Please, see Figures $3 \mathrm{C}$ and $3 \mathrm{D}$, where a clear difference in the recovery of flow can be observed in the two treatments; while flow after CA4 treatment exhibited a characteristic rebound, flow after G3-CA4 treatment remained depressed, indicating the continued effectiveness of the G3-CA4 treatment. The $\mathrm{T}_{2}$ (Figure 4C) and ADC (Figure 4D) maps show a ring of edema around a core of frank necrosis, signaled by a ring of increased $T_{2}$ and ADC [39]. Neither the edema (data not shown), nor the necrosis, were observable in the pre-treatment MRI. Similarly G3-succinamic acid vehicle treated MRI also did not show any sign of edema or necrosis (Figure 4A, a postcontrast $\mathrm{T}_{1}$-weighted image). The animals were sacrificed and perfused [40] and tumors were excised and further processed \& assessed using

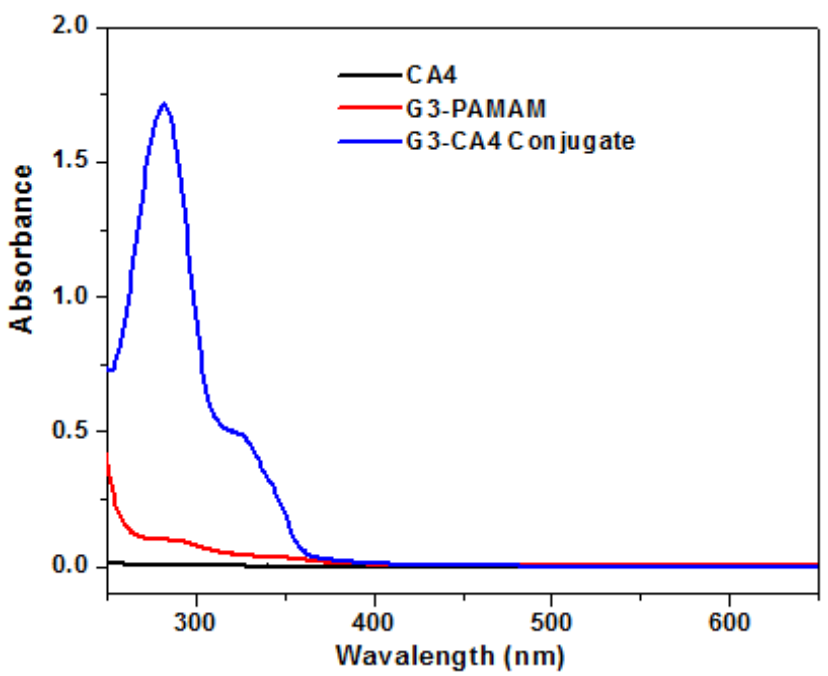

Figure 2: Absorption spectra of CA4 ( _ $)$ G3-PAMAM ( $\_$) and G3-CA4 conjugate $(-)$ in water.
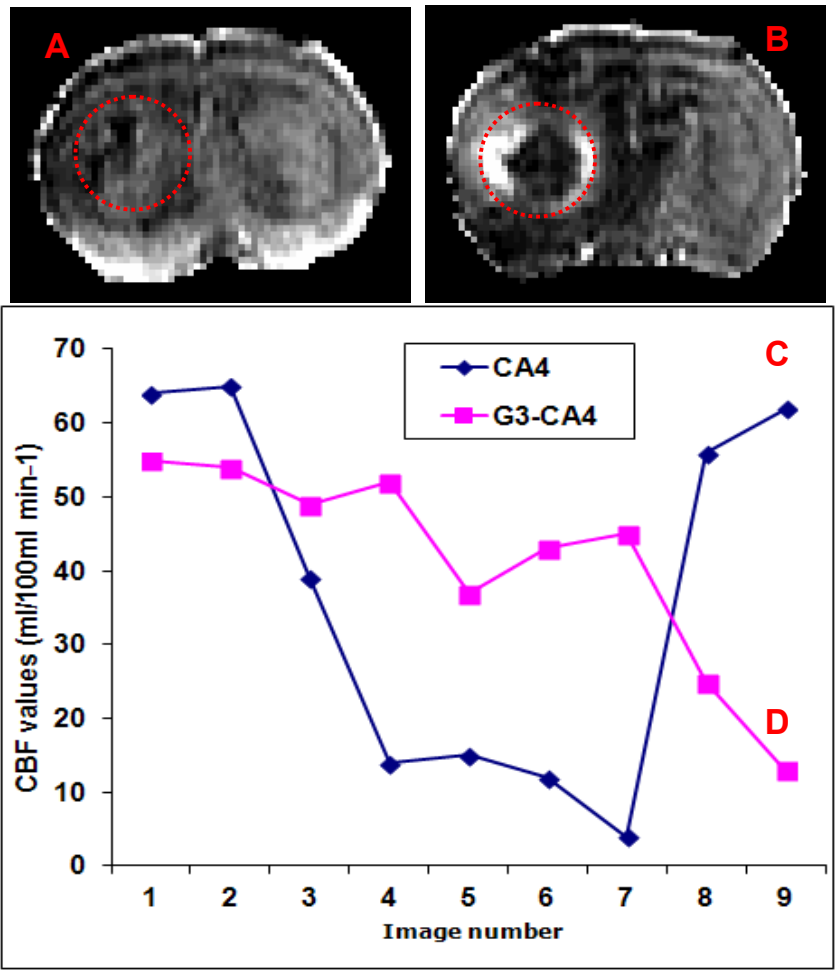

Figure 3: Representative CBF maps of CA4 (A) and G3-CA4 (B) treated rat brains bearing tumors after 24 hours post treatment. Blood flow vs time posttherapy in two nude rats with implanted U251 tumors. The first two points in each curve are flow pre-treatment. The last two points were taken $\sim 24$ $\mathrm{h}$ post-treatment. The five points following the first points were taken at $\sim 20$ min intervals. Blue curve (C) is flow post-CA4. The rapid decrease in flow, followed by a recovery at the $24 \mathrm{~h}$ point is evident. Pink curve (D) is flow postG3-CA4 treatment. The long-term effect of the latter treatment is evident in the depressed flow at the $24 \mathrm{~h}$ points. 
Citation: Gonawala S, Aryal M, Ewing JR, DeCarvaloh CA, Kalkanis S, et al. (2018) MRI Monitoring of Cerebral Blood Flow after the Delivery of Nanocombretastatin across the Blood Brain Tumor Barrier. J Nanomed Nanotechnol 9: 516. doi: 10.4172/2157-7439.1000516

Page 5 of 7

standard histopathology techniques. We investigated (by H\&E staining of tumor sections) the effect of G3-CA4 upon the morphology of the tumors (Figure 4E). A Large necrotic area is found together with destroyed blood vessels at the core of the tumor leaving viable tissue at the rim of the tumor (Figure $4 \mathrm{~F}$ and $4 \mathrm{G}$ ). Endothelial lining in the tumor is clearly delineated by FITC (green) labeled tomato lectin (Figure 4J) and the tumor peripheral active blood vessels (Figure $4 \mathrm{H}$ ) are stained by TRITC (red) labeled Von Willebrand factor (vWF). The nucleus of the cells is delineated by DAPI staining. Figure 4 (panels $\mathrm{H}$ to M) demonstrates the fluorescent microscopic images of tissue sections from tumor rim and core followed by G3-CA4 treatment. Use of vWF staining at the rim (Figure $4 \mathrm{H}$ ) and the core (Figure $4 \mathrm{~K}$ ) of the tumor has allowed us to assess changes in vessel numbers in G3-CA4 treated tumors. The vascular shutdown effect and subsequent intratumoral necrosis caused by i.v. delivery of G3-CA4 is clearly observed in U251 tumor. This demonstrates that a vascular bed responsive to G3-CA4 can be identified by a blood flow measurement.

The ineffectiveness of small molecule chemotherapy drugs in treating GBM has been attributed to the BBB being a significant impediment to the transvascular extravasation of drug fraction across the barrier into the extravascular compartment of tumor tissue. The overall ineffectiveness of these approaches can be attributed to the fact that there is only a transient elevation in drug concentrations within the extravascular extracellular compartment of tumor tissue due to the short blood half-life of small molecule chemotherapy [27]. We have previously developed highly water soluble dendrimerbased paramagnetic nanoparticles [26,41-43] and demonstrated the relative pharmacokinetics of generation G2 and G5 dendrimer-based paramagnetic nanoparticles within xenografts models by noninvasive MRI $[26,42]$. G2-based particle showed deeper tumor penetration while G5-based particle accumulated at the rim of the tumor first and then moved to the other parts of the tumor [41]. Like G3-CA4, we have also conjugated dietary supplement, curcumin (Cur), a yellow pigment in the spice turmeric (Curcuma longa) with a G3 succinamic acid dendrimer in our previous report [35]. Cur exhibits green fluorescence and has structural similarity with CA4. Both Cur and CA4 are natural product hydrophobic compounds. In addition, the G3-Cur conjugate has same size and surface charge as G3-CA4. While systemic delivery of Cur leads to non-specific distribution throughout the body [44], G3Cur conjugate preferentially accumulated in an orthotopic preclinical glioma model, minimizing systemic toxic effect [35]. An examination of tissue distribution in the same rat model showed that fluorescent labeled generation 3 (G3-Cur) was not present in major organs: heart, liver, spleen, lung and kidney [35]. We observed true success of delivering a significant amount of G3-Cur into brain tumor but not to the ipsilateral or contra-lateral normal brain regions. Thus, a G3-succinatecurcumin nanoparticle showed more accumulation in tumor and/ or less renal uptake, possibly as a result of small particle size, neutral
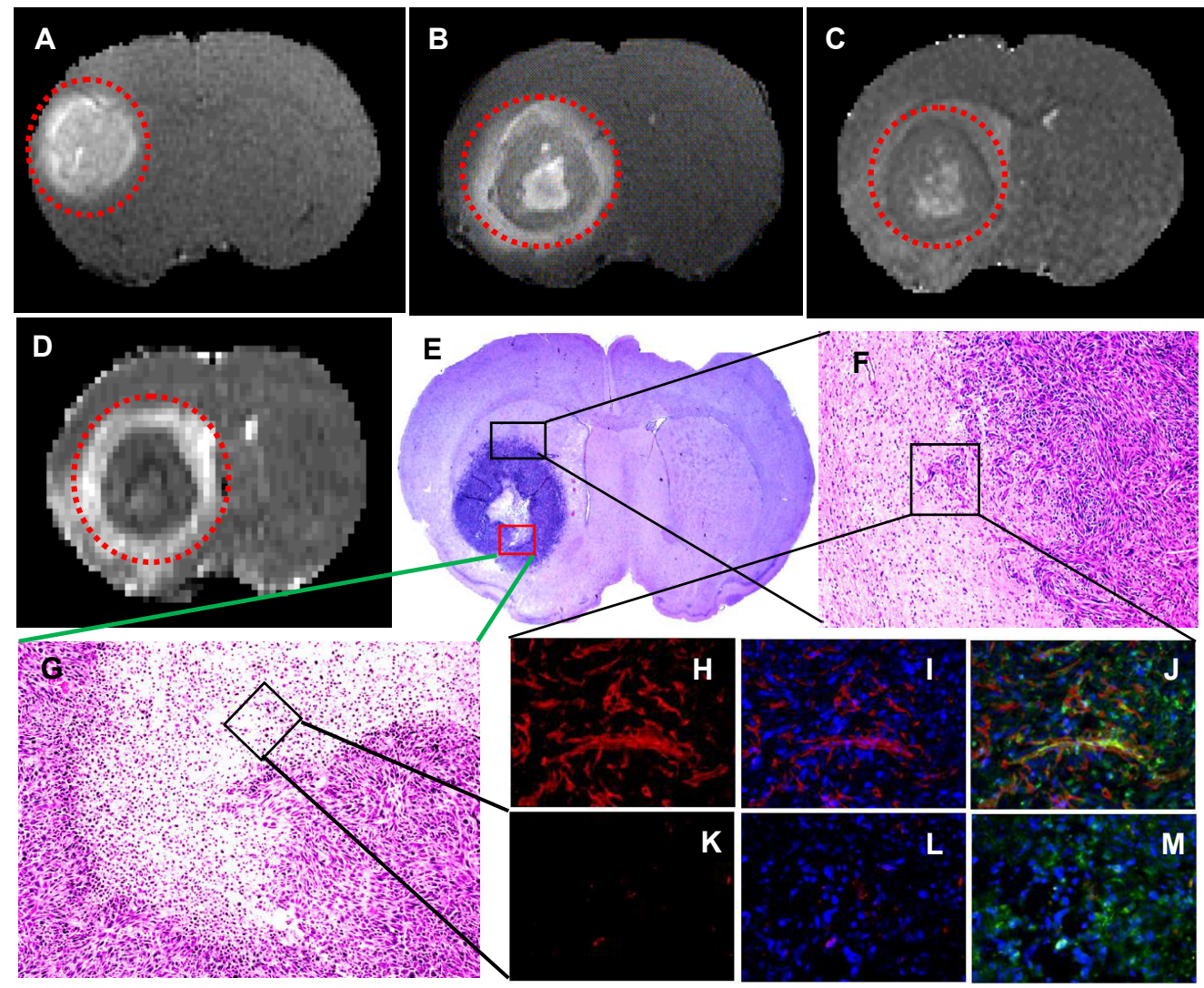

Figure 4: Post-contrast $T_{1}$-weighted MR images $24 \mathrm{~h}$ after administration of G3-succinamic acid polymer as vehicle (A) and G3-CA4 (B). $T_{2}(\mathrm{C})$ and $A D C$ maps (D) after $24 \mathrm{~h}$. Tumors are indicated as dotted red circles. Hematoxylin-eosin stain proves massive intratumoral necrosis (E \& $\mathrm{G}$ ) leaving viable tumor cells at the rim $(F)$. Representative images of tumor blood vessels (red visualized with vWF-TRITC staining) at panels $H, I$ \& at the rim. In contrast, the core of the tumor at panels K, L \& M did not show any active blood vessel. Red for vWF-TRITC and green for FITC-tomato lectin delineating endothelial lining and blue for nuclear dapi. 
Citation: Gonawala S, Aryal M, Ewing JR, DeCarvaloh CA, Kalkanis S, et al. (2018) MRI Monitoring of Cerebral Blood Flow after the Delivery of Nanocombretastatin across the Blood Brain Tumor Barrier. J Nanomed Nanotechnol 9: 516. doi: 10.4172/2157-7439.1000516

Page 6 of 7

surface charge and hydrophilicity in blood. We have conjugated 24 curcumin molecules with G3-succinate as fluorescent dye. Similarly, we have also conjugated 26 molecules of CA4 with G3- succinate polymer. Since, G3-Cur ${ }_{24}[35]$ and G3-(CA4) ${ }_{26}$ (Figure 1) are closely related in size, charge and structure, we expect that they also display very similar in vivo pharmacokinetics and biodistributions; we expect that G3-CA4 selectively accumulates at the U251 tumor site, avoiding off-target distribution. We have demonstrated that systemic delivery of G3-CA4 induced a permanent blood flow shut down, resulting in cellular death at the core. In contrast, CA4 depressed blood flow transiently with flow returning $24 \mathrm{~h}$ post treatment. Intravenous (i.v.) delivery of G3-CA4 in an orthotopic human glioma model caused necrosis at the core of the tumor, as validated by histo-pathlological studies (Figure 4). We have shown in our previous studies [40] that the U-251 glioma tumor exhibits relatively more expression of hypoxia inducible factor-1a (HIF-1 $\alpha$ ) at the core of the tumor than that of tumor periphery. It has been long known that the hypoxic microenvironment markedly affects the response of tumors to various treatments such as chemotherapy, radiotherapy and hyperthermia. Therefore, G3-(CA4) ${ }_{26}$ causes the destruction of large areas of the interior of brain tumors that are typically resistant to conventional chemo- and radio-therapies. By applying the designed nanoprodrug strategy and tumor-specific prodrug activation mechanism, we observed the first true success of inducing necrosis at the core of U-251 glioma tumor. We have demonstrated that a tumor vasculature disrupting approach is particularly suited for nanoparticle based therapeutics with a compromised blood brain barrier. PAMAM dendrimers of G7 and lower are generally used in human and animal systems as multifunctional carriers because they closely mimic the size of biomolecules [45]. However, PAMAM-based dendrimers are not considered to be biodegradable polymers; in particular, the G3-CA4 conjugate is not a biodegradable construct. However, once its potential is demonstrated, a CA4 conjugate with a biodegradable G3 polymer can be constructed for clinical translation.

\section{Conclusion}

Vascular disrupting agent, CA4 was conjugated with a G3succinamic acid polymer. Conjugation of CA4 with G3 polymer improved high water solubility as well as bioavailability. TEM images showed that the sizes of G3-(CA4) $)_{26}$ nanoparticles were in the range of 3 to $5 \mathrm{~nm}$, suggesting that the system could benefit from the EPR effect. The construct without CA4 conjugation had no therapeutic effect on glioma whereas, CA4 conjugated nanocombretastatin showed significant blood flow decrease in an experimental rat model of glioma. CA4 alone showed transient blood flow reduction, but the flow recovered after 24 hours. G3-(CA4) ${ }_{26}$ induced intratumoral blood vessels collapse leading to necrosis at the core of the tumor while the blood vessels at the periphery were alive. The delivery vehicle introduced here can be also loaded with other antiangiogenic drugs that can destroy active peripheral blood vessels, thus offering a possibility of combination therapy.

\section{Acknowledgements}

The authors acknowledge research support from the National Institutes of Health (NIH) grant RO1CA206190 to MMA.

\section{References}

1. Siegel R, Naishadham D, Jemal A (2012) Cancer statistics. CA Cancer J Clin 62: $10-29$.

2. Siegel R, DeSantis C, Virgo K, Stein K, Mariotto A, et al. (2012) Cancer treatment and survivorship statistics, 2012. CA Cancer J Clin 62: 220-241.

3. Johnson DR, O'Neill BP (2012) Glioblastoma survival in the United States before and during the temozolomide era. J Neurooncol 107: 359-364.
4. Dhermain F, Ducreux D, Bidault F, Bruna A, Parker F, et al. Use of the functional imaging modalities in radiation therapy treatment planning in patients with glioblastoma. Bull Cancer 92: 333-342.

5. Goldbrunner RH, Bendszus M, Wood J, Kiderlen M, Sasaki M, et al. (2004) PTK787/ZK222584, an inhibitor of vascular endothelial growth factor receptor tyrosine kinases, decreases glioma growth and vascularization. Neurosurgery 55: 426-432.

6. Norden AD, Young GS, Setayesh K, Muzikansky A, Klufas R, et al. (2008) Bevacizumab for recurrent malignant gliomas: efficacy, toxicity, and patterns of recurrence. Neurology 70: 779-787.

7. Sierra JR, Cepero V, Giordano S (2010) Molecular mechanisms of acquired resistance to tyrosine kinase targeted therapy. Mol Cancer 9: 75.

8. Norden AD, Drappatz J, Wen PY (2008) Novel anti-angiogenic therapies for malignant gliomas. Lancet Neurol 7: 1152-1160.

9. Verhoeff JJ, van Tellingen O, Claes A, Stalpers LJA, Linde ME, et al. (1999) Concerns about anti-angiogenic treatment in patients with glioblastoma multiforme. BMC Cancer 9: 444.

10. Ahluwalia MS, Gladson CL (2010) Progress on antiangiogenic therapy for patients with malignant glioma. J Oncol 2010: 689018.

11. Denekamp J (1982) Endothelial cell proliferation as a novel approach to targeting tumour therapy. $\mathrm{Br} \mathrm{J}$ Cancer 45: 136-139.

12. Denekamp J (1984) Vascular endothelium as the vulnerable element in tumours. Acta radiologica Oncology 23: 217-225.

13. Porcu E, Bortolozzi R, Basso G, Viola G (2014) Recent advances in vascular disrupting agents in cancer therapy. Future Med Chem 6: 1485-1498.

14. Siemann DW (2011) The unique characteristics of tumor vasculature and preclinical evidence for its selective disruption by Tumor-Vascular Disrupting Agents. Cancer Treat Rev 37: 63-74.

15. Galbraith SM, Chaplin DJ, Lee F, Stratford MR, Locke RJ, et al. (2001) Effects of combretastatin A4 phosphate on endothelial cell morphology in vitro and relationship to tumour vascular targeting activity in vivo. Anticancer Res 21 : 93-102.

16. Chaplin DJ, Dougherty GJ (1999) Tumour vasculature as a target for cancer therapy. Br J Cancer 80 1: 57-64.

17. Siemann DW, Chaplin DJ, Walicke PA (2009) A review and update of the current status of the vasculature-disabling agent combretastatin-A4 phosphate (CA4P). Expert Opin Investig Drugs 18: 189-197.

18. Vitale I, Antoccia A, Cenciarelli C, Crateri P, Meschini S, et al. (2007) Combretastatin CA-4 and combretastatin derivative induce mitotic catastrophe dependent on spindle checkpoint and caspase-3 activation in non-small cell lung cancer cells. Apoptosis 12: 155-166.

19. Lorusso PM, Boerner SA, Hunsberger S (2011) Clinical development of vascular disrupting agents: what lessons can we learn from ASA404? J Clin Oncol 29: 2952-2955.

20. Hinnen P, Eskens FALM (2007) Vascular disrupting agents in clinical development. Br J Cancer 96: 1159-1165.

21. Chaplin DJ, Pettit GR, Hill SA (1999) Anti-vascular approaches to solid tumour therapy: evaluation of combretastatin A4 phosphate. Anticancer Res 19: 189 195.

22. Vincent L, Kermani $P$, Young LM, Cheng J, Zhang F, et al. (2005) Combretastatin A4 phosphate induces rapid regression of tumor neovessels and growth through interference with vascular endothelial-cadherin signaling. $J$ Clin Invest 115: 2992-3006.

23. Tozer GM, Prise VE, Wilson J, Locke RJ, Vojnovic B, et al. (1999) Combretastatin A-4 phosphate as a tumor vascular-targeting agent: early effects in tumors and normal tissues. Cancer Res 59: 1626-1634.

24. Veiseh O, Sun C, Fang C, Bhattarai N, Gunn J, et al. (2009) Specific targeting of brain tumors with an optical/magnetic resonance imaging nanoprobe across the blood-brain barrier. Cancer Res 69: 6200-6207.

25. Jain R, Ellika SK, Scarpace L, Schultz LR, Rock JP, et al. (2008) Quantitative estimation of permeability surface-area product in astroglial brain tumors using perfusion CT and correlation with histopathologic grade. AJNR Am J Neuroradiol 29: 694-700. 
Citation: Gonawala S, Aryal M, Ewing JR, DeCarvaloh CA, Kalkanis S, et al. (2018) MRI Monitoring of Cerebral Blood Flow after the Delivery of Nanocombretastatin across the Blood Brain Tumor Barrier. J Nanomed Nanotechnol 9: 516. doi: 10.4172/2157-7439.1000516

26. Ali MM, Bhuiyan MP, Janic B, Varma NR, Mikkelsen T, et al. (2012) A nanosized PARACEST-fluorescence imaging contrast agent facilitates and validates in vivo CEST MRI detection of glioma. Nanomedicine (Lond) 7: 1827-1837.

27. Sarin H, Kanevsky AS, Wu H, Brimacombe KR, Fung SH, et al. (2008) Effective transvascular delivery of nanoparticles across the blood-brain tumor barrier into malignant glioma cells. J Transl Med 6: 80 .

28. Sarin H, Kanevsky AS, Wu H, Sousa AA, Wilson CM, et al. (2009) Physiologic upper limit of pore size in the blood-tumor barrier of malignant solid tumors. $J$ Transl Med 7: 51.

29. Sonavane G, Tomoda K, Makino K (2008) Biodistribution of colloidal gold nanoparticles after intravenous administration: effect of particle size. Colloids Surf B Biointerfaces 66: 274-280.

30. Binion DG, Otterson MF, Rafiee P (2008) Curcumin inhibits VEGF-mediated angiogenesis in human intestinal microvascular endothelial cells through COX2 and MAPK inhibition. Gut 57: 1509-1517.

31. Sarin H (2009) Recent progress towards development of effective systemic chemotherapy for the treatment of malignant brain tumors. J Transl Med 7: 77.

32. Sarin H (2010) Overcoming the challenges in the effective delivery of chemotherapies to CNS solid tumors. Ther Deliv 1: 289-305.

33. Karki K, Ewing JR, Ali MM (2016) Targeting Glioma with a Dual Mode Optical and Paramagnetic Nanoprobe across the Blood-brain Tumor Barrier. J Nanomed Nanotechnol 7: 395.

34. Venegas B, Zhu W, Haloupek NB, Lee J, Zellhart E, et al. (2012) Cholesterol superlattice modulates CA4P release from liposomes and CA4P cytotoxicity on mammary cancer cells. Biophys J 102: 2086-2094.

35. Gamage NH, Jing L, Worsham MJ, Ali MM (2016) Targeted Theranostic Approach for Glioma Using Dendrimer-Based Curcumin Nanoparticle. J Nanomed Nanotechnol 7: 393.

36. Ewing JR, Knight RA, Nagaraja TN, Yee JS, Nagesh V, et al. (2003) Patlak plots of Gd-DTPA MRI data yield blood-brain transfer constants concordant with those of $14 \mathrm{C}$-sucrose in areas of blood-brain opening. Magn Reson Med 50: 283-292.

37. Sumer Bolu B, Manavoglu Gecici E, Sanyal R (2016) Combretastatin A-4 Conjugated Antiangiogenic Micellar Drug Delivery Systems Using DendronPolymer Conjugates. Mol Pharmaceutics 13: 1482-1490.

38. Zhang M, Guo R, Wang Y, Cao X, Shen M, et al. (2011) Multifunctional dendrimer/combretastatin A4 inclusion complexes enable in vitro targeted cancer therapy. Int J Nanomedicine 6: 2337-2349.

39. Junjie Li, Feng Chen, Yuanbo Feng, Marlein Miranda Cona, Jie Yu, et al. (2013) Diverse responses to vascular disrupting agent combretastatin a4 phosphate: a comparative study in rats with hepatic and subcutaneous tumor allografts using MRI biomarkers, microangiography, and histopathology. Transl Oncol 6: 42-50.

40. Ali MM, Janic B, Babajani-Feremi A, Varma NR, Iskander AS, et al. (2010) Changes in vascular permeability and expression of different angiogenic factors following anti-angiogenic treatment in rat glioma. PloS one 5: e8727.

41. Ali MM, Liu G, Shah T, Flask CA, Pagel MD (2009) Using two chemical exchange saturation transfer magnetic resonance imaging contrast agents for molecular imaging studies. Acc Chem Res 42: 915-924.

42. Ali MM, Yoo B, Pagel MD (2009) Tracking the relative in vivo pharmacokinetics of nanoparticles with PARACEST MRI. Mol Pharm 6: 1409-1416.

43. Ali MM, Woods M, Caravan P, Opina AC, Spiller M, et al. (2008) Synthesis and relaxometric studies of a dendrimer-based $\mathrm{pH}$-responsive MRI contrast agent. Chemistry 14: 7250-7258.

44. Yallapu MM, Othman SF, Curtis ET, Bauer NA, Chauhan N, et al. (2012) Curcumin-loaded magnetic nanoparticles for breast cancer therapeutics and imaging applications. Int J Nanomedicine 7: 1761-1779.

45. Islam MT, Majoros IJ, Baker JR (2005) HPLC analysis of PAMAM dendrimer based multifunctional devices. J Chromatogr B Analyt Technol Biomed Life Sc 822: 21-26. 